\title{
A case of massive splenomegaly due to chronic myeloproliferative neoplasm
}

\author{
Zachary G. Jacobs ${ }^{1}$, Bongani Kaimila ${ }^{2}$, Peter M. Wasswa ${ }^{3}$, Thuy Bui ${ }^{4}$ \\ 1. Department of Medicine, University of Pittsburgh, Pittsburgh, PA \\ 2. University of North Carolina Project-Malawi, Lilongwe, Malawi \\ 3. Baylor College, Lilongwe Malawi \\ 4. Director, Global Health and Underserved Populations Track, University of Pittsburgh Medical Center \\ Internal Medicine Residency Training Program
}

Corresponding author: Zachary G. Jacobs; Zachary.Jacobs@ucsf.edu

\section{Case Presentantion}

A 34-year-old female, HIV non-reactive, presented to Kamuzu Central Hospital $(\mathrm{KCH})$ in Lilongwe, Malawi, complaining of 7 months abdominal pain. She endorsed shortness of breath and early satiety for the same duration, but denied fever, chills, night sweats, and weight loss. The remainder of her review of systems was negative. She had no significant past medical history. There were no identifiable risk factors for myeloproliferative disorders, including family history, known genetic syndromes, chemical exposure, or history of ionizing radiation. On physical exam, she was noted to have a visible spleen (figure 1) which crossed into the right upper quadrant as well as the pelvis, and was non-tender to palpation. There was no cervical or axillary lymphadenopathy. The remainder of her exam was unremarkable.
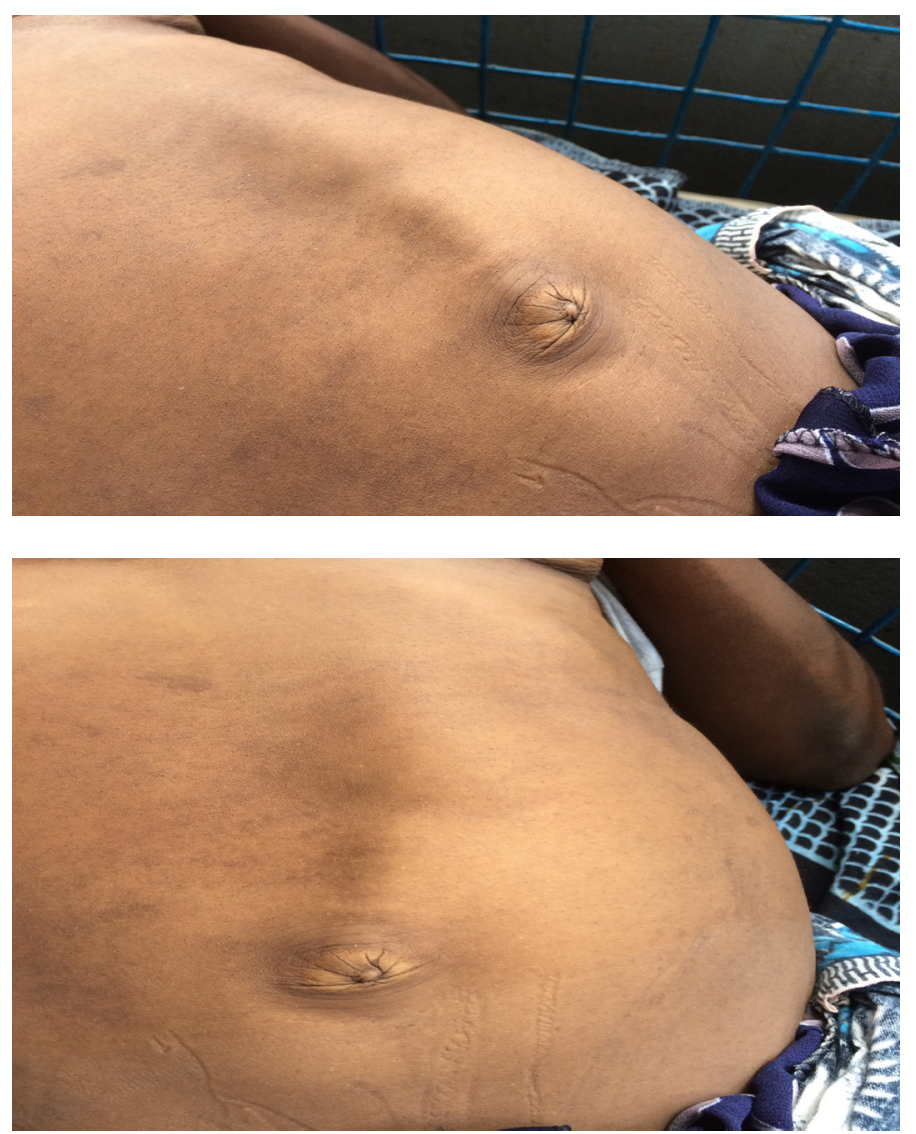

Figure 1: photographs of the patient's massively enlarged spleen at the time of admission, which crossed the midline and extended into the pelvis.
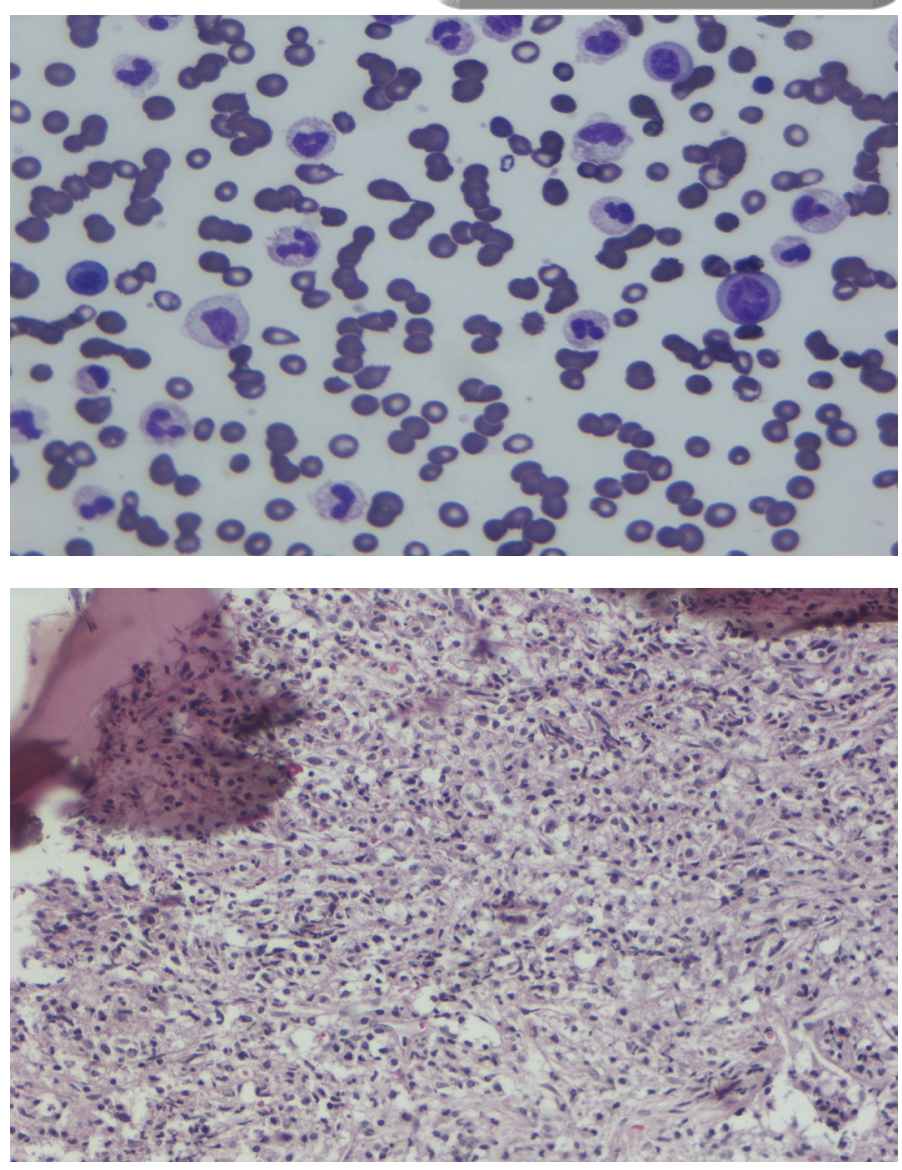

Figure 2: peripheral blood film demonstrating neutrophilia $(53 \%$ of nucleated cells), $22 \%$ myelocytes, $14 \%$ band cells, $1 \%$ myeloblasts, and very occasional nucleated red blood cells. No evidence of basophilia or dysplasia

An abdominal ultrasound revealed $16 \mathrm{~cm}$ homogenous liver, normal kidneys, with no free fluid or masses in the abdomen, and a $1.3 \mathrm{~cm}$ pericardial effusion, and a large spleen with no comment on specific dimensions.

Her white blood cell (WBC) count was 94 x109 cells/L (ref. range: 4-10), with 89\% neutrophils (ref. range: $27-60$ ), 7\% lymphocytes (ref. range: 29-59), and 4\% monocytes (ref. range: 2-10). Haemoglobin was $5.4 \mathrm{~g} / \mathrm{dL}$ (ref. range: 10.917.3) with a mean corpuscular volume (MCV) of $116 \mathrm{fL}$ (ref. range: 71-95), and platelet count was 119 x109 cells/L (ref. range: 122-330). A peripheral blood film was reviewed by the staff haematologist and interpreted as neutrophilia with bandaemia, and myelocytosis with 1\% myeloblasts (figure 2). Erythrocyte sedimentation rate (ESR) was $40 \mathrm{~mm} / \mathrm{hr}$ (ref. 
range: $0-15)$.

The patient underwent bone marrow biopsy, which demonstrated hyper-cellularity along with extensive fibrosis, which masked interpretation of the underlying morphology. The combination of findings was interpreted by the local haemato-pathologist as a myeloproliferative neoplasm, possibly chronic myeloid leukaemia (CML). Unfortunately, confirmatory genetic testing is not presently available in Malawi, and coordinating referral to locations able to do so (e.g. South Africa) proves challenging. Only those patients who test positive for BCR-ABL are eligible for treatment given limited drug supply; so as of now the patient remains untreated.

Table I: Differential diagnosis of massive splenomegaly ${ }^{2}$

Myeloproliferative disorders:

Chronic myeloid leukaemia

Myelofibrosis

Polycythemia vera

Essential thrombocytosis

Lymphoma

Gaucher's disease

Thalassemia major

Visceral leishmaniasis (kala-azar)

Hyper-reactive malarial splenomegaly (HMS)

AIDS with disseminated Mycobacterium-avium Complex (MAC)

\section{Discussion}

Here we present a case of a 34-year-old female, HIV nonreactive, with no significant past medical history, who presented with a massively enlarged spleen and was found to have chronic myeloproliferative neoplasm most likely due to CML.

Splenomegaly is a common physical exam finding, particularly in Africa, which is associated with an extensive list of diseases including infectious, haematologic, congestive, inflammatory, and neoplastic aetiologies, just to name a few ${ }^{1}$. On the other hand, massive splenomegaly, typically defined as a spleen which extends into the pelvis, or crosses the midline, has a much narrower differential diagnosis $^{2}$. Causes of a massively enlarged spleen include myeloproliferative disorders (such as chronic myeloid leukaemia, polycythemia vera, primary myelofibrosis, and essential thrombocytosis), Gaucher's disease, lymphoma (typically indolent), thalassemia major,visceral leishmaniasis (kala-azar) and hyper-reactive malarial splenomegaly (HMS), formerly known as tropical splenomegaly syndrome (see Table I). AIDS with disseminated Mycobacterium avium complex (MAC) infection has also been recognized in at least one case series as a potential cause of a massively enlarged spleen $^{3}$. Other conditions, which are commonly associated with sub-massive splenomegaly, such as portal hypertension, hemolytic anemias, and other infectious aetiologies, will rarely progress to massive splenomegaly ${ }^{3}$.

While the exact mechanisms leading to hypersplenism vary somewhat based on the causative aetiology, the underlying pathophysiologic changes are the same, including splenic congestion and hyper-functioning/hypertrophy ${ }^{4}$. In the case of myeloproliferative disorders, this is due to a combination of 1) pooling of higher numbers of circulating red blood cells; and 2) extramedullary haemopoeisis (also known as myeloid metaplasia), which leads to an expansion of splenic reticular elements.

In sub-Saharan Africa, massive splenomegaly is frequently reported as quite common ${ }^{5,6}$, though actual prevalence estimates are hard to come by ${ }^{7}$. In malaria-endemic countries, HMS is typically cited as a leading cause of massive splenomegaly ${ }^{5}$. In a case series of 221 Ghanaian patients published in 2002, massive splenomegaly was associated with HMS in $41 \%$ of cases, while B-lymphoproliferative disorders came in second, accounting for $22 \%$ of cases; of the remaining cases, $14 \%$ had other haematologic malignancies, and $23 \%$ had no identifiable cause ${ }^{7}$.

Splenomegaly is an important physical exam finding to recognize, particularly when the spleen is massively enlarged. Since the differential diagnosis is limited to the items presented in table I, it is easy to tailor the initial diagnostic workup of these cases. First-pass testing will vary based on history, exam, and clinical features, which may point toward infectious, neoplastic, or other aetiologies. Availability of resources needs to be considered, as do the epidemiologic features of these diseases (for example, leishmaniasis is rare in Malawi, and Gaucher's is rare in adults worldwide). Many causes of massive splenomegaly will present with abnormalities in one or more cell lines. For example, CML, along with other myeloproliferative disorders and lymphomas, commonly present with elevated WBC, often with atypical cells; leishmaniasis and Gaucher's disease both often cause variable pancytopenia, whereas a profound microcytic anemia could be suggestive of thalassemia major ${ }^{2}$. Keeping this in mind, a reasonable first-pass diagnostic workup for massive splenomegaly could include full blood counts, peripheral blood smear, HIV testing and, depending on the results of these tests, potentially a bone marrow biopsy.

\section{Take Home Points}

1.Splenomegaly is a common physical exam finding, especially in Sub-Saharan Africa

2.Massive splenomegaly is defined as a spleen extending into the pelvis or crossing the midline

3.Massive splenomegaly has a limited differential diagnosis (table I); workup should be tailored according to local epidemiology and pre-test probability

\section{References}

1. Pozo AL, Godfrey EM, Bowles KM. Splenomegaly: Investigation, diagnosis and management. Blood rev. Journal 2009; 23(3): 105-111.

2. Luo EJ, Levitt L. Massive splenomegaly. Hosp Physician. 2008; 44: 31-38

3. O'Reilly RA. Splenomegaly in 2,505 patients at a large university medical center from 1913 to 1995. 1963 to 1995: 449 patients. West J Med. 1998; 69(2): 88-97.

4. Zhang B \& Lewis SM. The splenomegaly of myeloproliferative and lymphoproliferative disorders: Splenic cellularity and vascularity. Eur J Haematol. 1989; 43: 63-66 
5. Leoni S, Buonfrate D, Angheben A, Gobbi F, Bisoffi Z. The hyper- 7. Bedu-Addo G, Bates I. Causes of massive tropical splenomegaly in reactive malarial splenomegaly: a systematic review of the literature. Ghana. Lancet. 2002; 360 (9331): 449-454. Malar J. 2015; 14: 185.

6. Lowenthal MN, Hutt MS, Jones IG, Mohelsky V, O’Riordan EC. Massive splenomegaly in Northern Zambia. I. Analysis of 344 cases. Trans R Soc Trop Med Hyg. 1980; 74(1): 91-8. 